\section{Causes of Hospital Mortality in Patients with Rheumatoid Arthritis and Systemic Lupus Erythematosus in a University Hospital: 1998-2015 Analysis}

\section{To the Editor:}

With great interest we read the article entitled "Hospitalizations in Patients with Systemic Lupus Erythematosus in an Academic Health Science Center" by Gu, et $a l^{1}$. They described the hospitalization of patients with systemic lupus erythematosus (SLE) in an academic hospital over 2- and 3-year periods. They found that incidental causes were most common (35.6\%), and that $21.4 \%$ and $22.4 \%$ of admissions were because of active SLE and infection, respectively. We made a retrospective analysis of 1330 medical records regarding hospitalization of SLE or rheumatoid arthritis (RA) patients in an 18-year period. Table 1 shows the frequency of hospitalization per year, and Table 2 shows the all-cause mortality trends. Of the 467 RA hospital admissions, $5.1 \%$ of the patients died, and for the 863 SLE hospital admissions, $22.1 \%$ died. In a subgroup analysis in 2008-2009, we found that the mortality-associated factors corresponded to dyspnea as the chief complaint symptom at admission (OR 5.8, 95\% CI 1.6-14, $\mathrm{p}=0.002)$ and intensive care admission (OR 9.3, 95\% CI 3-29, p = 0.04). Three or more office/control consultations were associated with less mortality (OR 0.59 , 95\% CI 0.5-0.9, p = 0.03). Advances in the diagnosis and treatment of SLE and RA have reduced the morbidity and mortality of both diseases. Infectious and cardiovascular pathologies were the most frequent causes of death. Considering these factors and the ones addressed by Gu K, et al is of great importance in an academic/university field.

DAVID VEGA-MORALES, MD, MSc, PhD, IVAN DE JESÚS HERNÁNDEZ-GALARZA, MD, DIONICIO ÁNGEL

GALARZA-DELGADO, MD, PhD, Universidad Autónoma de Nuevo León, Hospital Universitario Dr. José Eleuterio González, Rheumatology Service, Internal Medicine Department, Monterrey, Nuevo León, Mexico.

Address correspondence to Dr. D. Vega-Morales, Hospital Universitario, Rheumatology, Francisco I. Madero S/N, Mitras Centro, Monterrey,

Nuevo León 64460, Mexico. E-mail: drdavidvega@yahoo.com.mx

\section{REFERENCE}

1. Gu K, Gladman DD, Su J, Urowitz MB. Hospitalizations in patients with systemic lupus erythematosus in an academic health science center. J Rheumatol 2017;44:1173-8.

J Rheumatol 2018;45:4; doi:10.3899/jrheum.170735

Table 1. Hospital admissions and deaths per year. Data are $\mathrm{n}(\%)$.

\begin{tabular}{ccccc}
\hline & \multicolumn{2}{c}{ Rheumatoid Arthritis } & \multicolumn{2}{c}{ Systemic Lupus Erythematosus } \\
& Hospital Admissions, & Deaths, & Hospital Admissions, & Deaths, \\
$\mathrm{n}=467$ & $\mathrm{n}=24$ & $\mathrm{n}=863$ & $\mathrm{n}=191$ \\
\hline 1998 & $6(1.3)$ & $0(0)$ & $19(2.2)$ & $6(31.6)$ \\
1999 & $12(2.6)$ & $3(25)$ & $38(4.4)$ & $6(15.8)$ \\
2000 & $22(4.7)$ & $0(0)$ & $21(2.4)$ & $4(19)$ \\
2001 & $14(3)$ & $0(0)$ & $36(4.2)$ & $5(13.9)$ \\
2002 & $17(3.6)$ & $0(0)$ & $22(2.5)$ & $2(9.1)$ \\
2003 & $26(5.6)$ & $0(0)$ & $65(7.5)$ & $22(33.8)$ \\
2004 & $33(7.1)$ & $1(3.03)$ & $40(4.6)$ & $9(22.5)$ \\
2005 & $28(6)$ & $1(3.57)$ & $64(7.4)$ & $32(50)$ \\
2006 & $27(5.8)$ & $0(0)$ & $39(4.5)$ & $7(17.9)$ \\
2007 & $34(7.3)$ & $0(0)$ & $33(3.8)$ & $9(27.3)$ \\
2008 & $40(8.6)$ & $2(5)$ & $49(5.6)$ & $19(38.8)$ \\
2009 & $28(6)$ & $0(0)$ & $53(6.1)$ & $8(15.1)$ \\
2010 & $24(5.1)$ & $7(29.17)$ & $60(7)$ & $10(16.7)$ \\
2011 & $27(5.8)$ & $0(0)$ & $51(5.9)$ & $9(17.6)$ \\
2012 & $31(6.6)$ & $2(6.45)$ & $80(9.3)$ & $9(11.3)$ \\
2013 & $41(8.8)$ & $6(14.63)$ & $88(10.2)$ & $15(17)$ \\
2014 & $40(8.6)$ & $2(5)$ & $66(7.6)$ & $16(24.2)$ \\
2015 & $17(3.6)$ & $0(0)$ & $39(4.5)$ & $3(7.7)$ \\
\hline
\end{tabular}


Table 2. Mortality causes in rheumatoid arthritis (RA) and systemic lupus erythematosus (SLE). Data are n (\%).

\begin{tabular}{lcc}
\hline Etiology & RA, $\mathrm{n}=24$ & SLE, $\mathrm{n}=191$ \\
\hline Acute respiratory failure & $7(29.1)$ & $46(24)$ \\
Sepsis, unspecified & $6(25)$ & $39(20.4)$ \\
Other sudden death, cause unknown & $4(16.6)$ & $27(14.1)$ \\
Alveolar and parietoalveolar conditions & $0(0)$ & $12(6.2)$ \\
Cardiac conduction disorder, unspecified & $0(0)$ & $9(4.7)$ \\
Systemic inflammatory response syndrome of infectious & & \\
$\quad$ origin with organ failure & $0(0)$ & $8(4.1)$ \\
Cardiogenic shock & $1(4.1)$ & $7(3.6)$ \\
Acidosis & $0(0)$ & $4(2)$ \\
Pneumonia, unspecified & $0(0)$ & $4(2)$ \\
Hypovolemic shock & $0(0)$ & $4(2)$ \\
Bacterial meningitis, unspecified & $0(0)$ & $3(1.5)$ \\
Disorder of central nervous system, unspecified & $0(0)$ & $3(1.5)$ \\
Endocarditis, valve unspecified & $0(0)$ & $3(1.5)$ \\
Pneumonitis from food and vomit & $0(0)$ & $3(1.5)$ \\
Chronic kidney disease & $0(0)$ & $3(1.5)$ \\
Histoplasmosis, unspecified & $0(0)$ & $2(1)$ \\
Other lipid storage disorders & $0(0)$ & $2(1)$ \\
Cardiac arrhythmia, unspecified & $1(4.1)$ & $2(1)$ \\
Cerebrovascular diseases & $2(8.3)$ & $2(1)$ \\
Thoracoabdominal aortic aneurysm, without mention of rupture & $0(0)$ & $2(1)$ \\
Pulmonary edema & $0(0)$ & $2(1)$ \\
Other diseases of jaws & $0(0)$ & $2(1)$ \\
Dilated cardiomyopathy & $0(0)$ & $1(0.5)$ \\
Hypertensive encephalopathy & $0(0)$ & $1(0.5)$ \\
Interstitial pulmonary disease, unspecified & $1(4.1)$ & $0(0)$ \\
Cervical disc disorders & $2(8.3)$ & $0(0)$ \\
\hline
\end{tabular}

